\title{
Development of a Heat-Driven-Type MEMS Olfactory Display ${ }^{\dagger}$
}

\author{
Hiroki Ishizuka ${ }^{1, *}$, Kenta Kashiwagi ${ }^{2}$, Kyohei Terao ${ }^{1}$, Hidekuni Takao ${ }^{1}$ and Fusao Shimokawa ${ }^{1}$ \\ 1 Area in Mechanical Systems Engineering, Faculty of Engineering and Design, Kagawa University, Kagawa \\ 761-0396, Japan; terao@eng.kagawa-u.ac.jp (K.T.); takao@eng.kagawa-u.ac.jp (H.T.); \\ simokawa@eng.kagawa-u.ac.jp (F.S.) \\ 2 RICOH, Tokyo 143-8555, Japan; kenta.kashiwagi@jp.ricoh.com \\ * Correspondence: hi1124@eng.kagawa-u.ac.jp; Tel.: +81-87-864-2343 \\ + Presented at the Eurosensors 2018 Conference, Graz, Austria, 9-12 September 2018.
}

Published: 26 December 2018

\begin{abstract}
An olfactory display is necessary for effective multimodal information communication. The relatively large size of current olfactory displays does not support integration with other information devices. Thus, in this study, a heat-driven-type microelectromechanical system (MEMS) olfactory display that is only a few square centimeters in size and, thus, suitable for integration, is proposed. The olfactory display was fabricated by implementing a microfabrication process, and the fundamental driving conditions for the heater and valve were confirmed. Furthermore, a perfume diffusion experiment was conducted to characterize the olfactory display. The diffusion of the perfume was successfully synchronized with the open/close time of the valve.
\end{abstract}

Keywords: olfactory display; MEMS; actuator; human interface

\section{Introduction}

To realize multimodal information communication, an olfactory display, which provides olfactory information to users, has been developed by researchers and in the industry. For example, Hasegawa et al. developed an olfactory display using an array of ultrasound transducers [1]. The olfactory display can control the diffusion of perfume with ultrasonic radiation and selectively provide the perfume to the target users. Dobbelstein et al. developed a wearable-type olfactory display [2]. The olfactory display can provide eight types of olfactory information with the vaporization of perfumes. Narumi et al. developed a system to add olfactory information to visual information [3]. They experimentally showed that olfactory information and visual information have an interaction. For example, the taste of cookies can be modulated with olfactory information and visual information. The sizes of the olfactory displays mentioned above are too large. Thus, these olfactory displays are not suitable to be integrated into other information devices to add olfactory information to other information.

A miniature olfactory display with a microfabrication process is proposed. The size of the proposed display is a few square centimeters. The olfactory display consists of a heating part and a chamber with a mechanical valve to store perfumes. The stored perfume is vaporized via a heater, and the diffusion of the perfumes is enabled/disabled via a microvalve. The diffused volume of the perfume is determined by the power of the heater and the open/close time of the valve. The structure and principle of the olfactory display can be easily miniaturized and arrayed densely on a substrate. Therefore, the olfactory display has the potential to be integrated into other devices to add various olfactory information to other information. In this study, the size of the olfactory display was 
determined, and it was fabricated. An experiment was conducted to evaluate the perfume diffusion performance.

\section{Principle}

Figure 1 shows the principle of the proposed olfactory display. The olfactory display consists of a heater, a chamber to store perfumes, and a mechanical valve. They are formed on a substrate. The heat generated by the heater is conducted to the chamber through the substrate. The valve is based on an electrostatic force. Without voltage to the heater and the valve, perfume is not diffused, because the perfume is perfectly sealed into the chamber. With voltage, the perfume is vaporized by the heater. Then, the valve is opened, and the vaporized perfume is diffused. The amount and intensity of the vaporized perfume are controlled by the heating time and the valve's opening time.

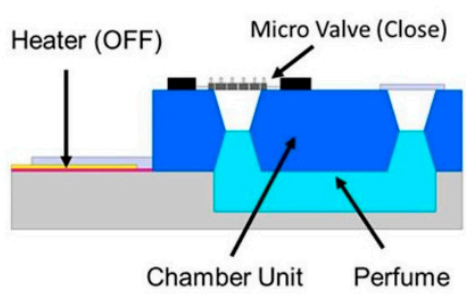

(a)

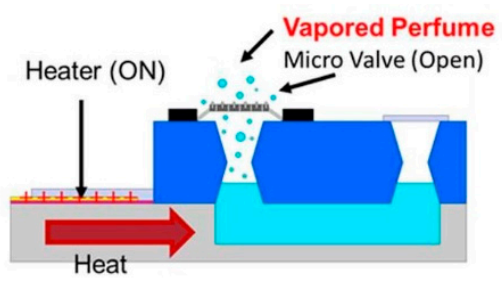

(b)

Figure 1. Basic principle of the proposed olfactory display. (a) Heater and value in off and closed states, respectively; and (b) Heater and value in on and open states, respectively.

\section{Design}

The valve consisted of a moving part and a fixed part. With voltage, the moving part is attracted toward the fixed part. Then, the valve is opened. The designed displacement of the actuator was 8 $\mu \mathrm{m}$, and the diameter of the valve was $3 \mathrm{~mm}$. The entire size of the valve part was $1 \mathrm{~cm} \times 1 \mathrm{~cm}$. The valve was formed on a silicon substrate with a microfabrication process. The photograph of the fabricated valve is shown in Figure 2a.

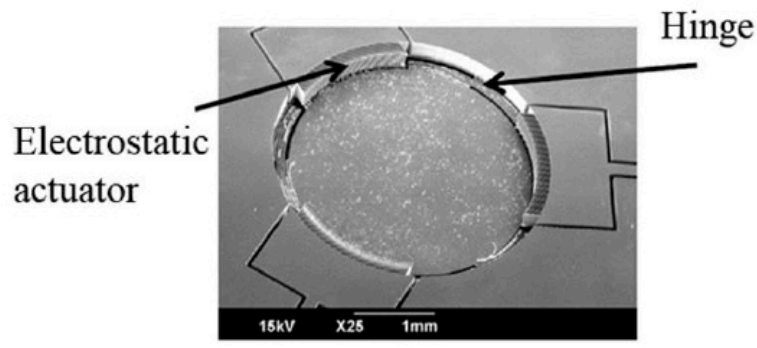

(a)

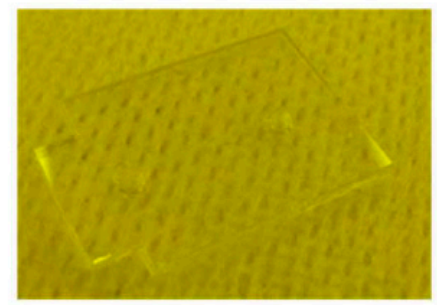

(c)

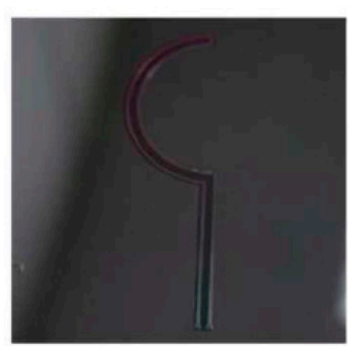

(b)

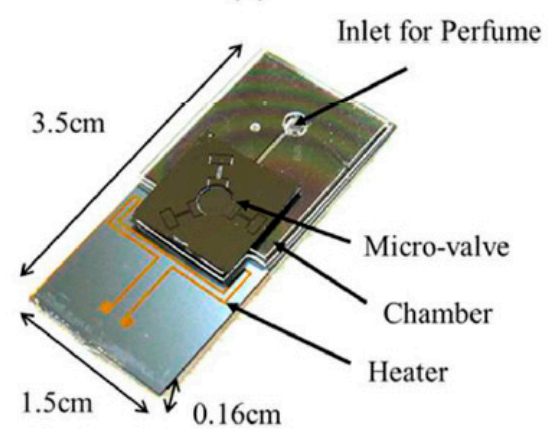

(d)

Figure 2. Photograph of the fabricated components. (a) The value. (b) The heater. (c) The chamber. (d) The integrated olfactory display. 
The heater consisted of a metal wire. The requirement of the heater was heating perfumes at 100 ${ }^{\circ} \mathrm{C}$. The wire was designed to satisfy the requirement. The resulting dimensions of the wire were 20$\mu \mathrm{m}$ width and 12-mm length. The heater was fabricated on a silicon substrate with Ti deposition and etching. Figure $2 b$ shows the fabricated heater.

The chamber was formed by etching a glass plate. The chamber was cylindrical. The diameter of the chamber was $1.5 \mathrm{~mm}$, and the height was $1 \mathrm{~mm}$. The fabricated chamber is shown in Figure 2c.

The valve, the heater, and the chamber were integrated with anodic bonding. Figure $2 \mathrm{~d}$ shows a photograph of the integrated olfactory display. The total size of the completed olfactory display was $1.5 \mathrm{~cm} \times 3.5 \mathrm{~cm} \times 0.16 \mathrm{~mm}$.

\section{Experimental Procedure}

An experiment was conducted to characterize the fabricated olfactory display. Figure 3 shows the experimental setup. The experimental setup consisted of an olfactory display, a gas sensor (Figaro TGS822), and a sealed container. To evaluate the perfume diffusion, ethanol was used as a perfume. Ethanol was mixed with triacetin to prevent vaporization and then injected into the chamber with a pipette and used as a perfume. In the experiment, the characteristics of the heater were evaluated. The relationship between the applied voltage and temperature of heater was measured by using a noncontact temperature sensor. The voltage was varied from $0 \mathrm{~V}$ to $60 \mathrm{~V}$. The relationship between the applied voltage and displacement of valve was evaluated by using a microscope. The voltage was varied from $0 \mathrm{~V}$ to $80 \mathrm{~V}$. Then, it was evaluated how the open time of the valve affected the diffusion by varying the time that the valve remained in an open state between $1 \mathrm{~s}$ and $5 \mathrm{~s}$.

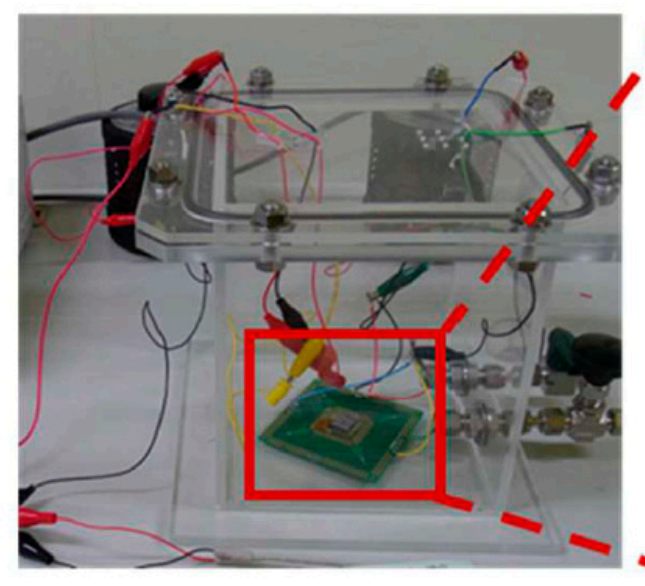

(a)

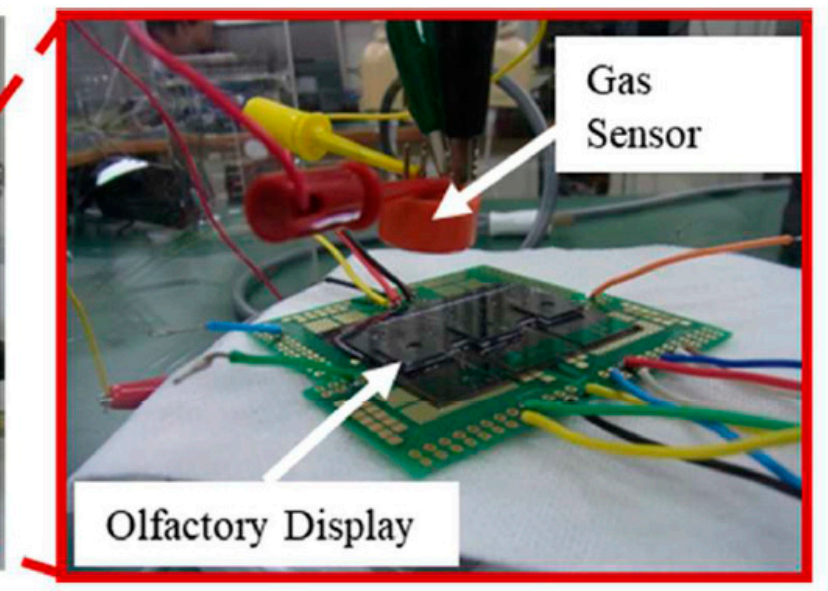

(b)

Figure 3. Experimental setup for diffusion experiment. (a) The olfactory display was placed in a sealed container. (b) A gas sensor was used to measure the diffusion.

\section{Experimental Results}

\subsection{Characterization of Heater and Valve}

Figure 4 a shows the results for the heater. The temperature increased with the increase in the applied voltage. The maximum temperature was $90^{\circ} \mathrm{C}$. The results indicate that the temperature of the heater can be controlled by the applied voltage. However, the range of the temperature was almost $60^{\circ} \mathrm{C}$ and not sufficiently wide. Therefore, the fabricated heater can be only applied to vaporize perfumes that have a relatively low boiling point. Figure $4 \mathrm{~b}$ shows the results for the valve. The displacement of the valve linearly increased with the increase in the voltage. To open the valve, almost $20 \mathrm{~V}$ was minimally required. The displacement of the valve reached $8 \mu \mathrm{m}$ at $80 \mathrm{~V}$. The results indicate that the perfume diffusion can be controlled by controlling the amplitude of the applied 
voltage. At $80 \mathrm{~V}$, the valve was not broken by the applied voltage. Thus, the following experiment was conducted by applying a voltage of $80 \mathrm{~V}$ to maximize the displacement of the valve.

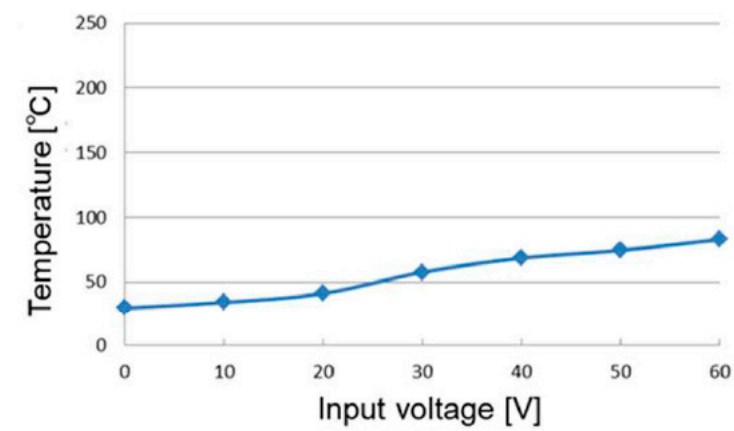

(a)

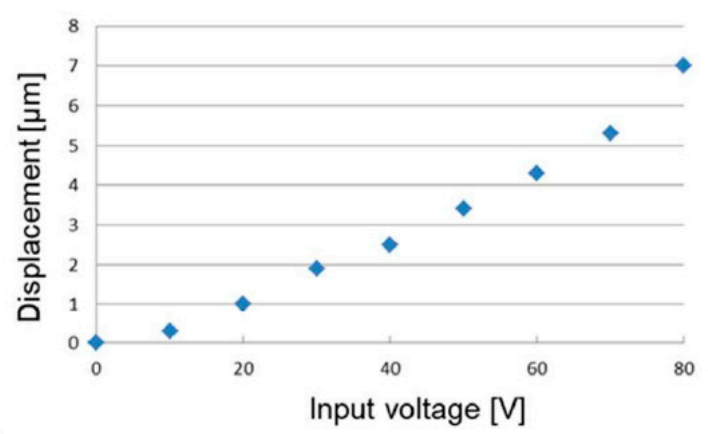

(b)

Figure 4. Experimental results. (a) Relationship between voltage and temperature. (b) Relationship between voltage and displacement.

\subsection{Characterization of Heater and Valve}

Figure 5 shows the experimental results for the characterization of the olfactory display. The gas sensor successfully detected the diffusion of the perfume when the heater and valve were in their ON and open states, respectively. The duration of the diffusion was found to increase with the increase of the time that the valve remained in its open state, and diffusion was confirmed to be terminated when the valve was switched to a closed state. The perfume was diffused immediately after the heater and valve were in their $\mathrm{ON}$ and open states. However, the diffusion did not immediately stop after the heater and valve were in their OFF and closed states. To control the perfume diffusion with high accuracy, the substantivity of the perfume should be considered.

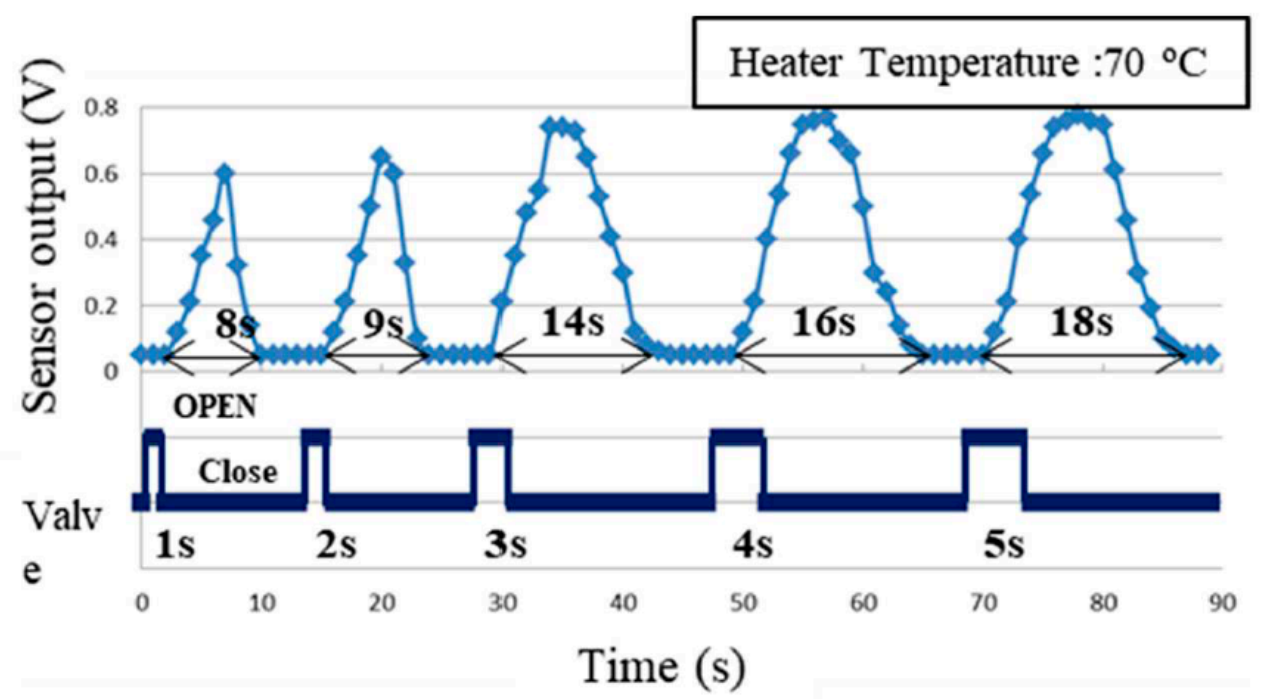

Figure 5. Relationship between the time and the sensor output. The open time of the value was varied to evaluate the diffusion of the vapored perfume. The diffusion was successfully controlled by the open time.

\section{Conclusions}

In this study, a miniature olfactory display was designed and fabricated that is sufficiently small to be integrated into other devices. The fabricated olfactory display was characterized. The experimental results indicate that the olfactory display has the potential to provide olfactory information. For the next study, experiments with perfumes are planned. Also, methods to control the perfume diffusion with high accuracy will be considered. 
Author Contributions: K.K. designed and performed the experiments; K.K. fabricated the device. H.I. and K.K. analyzed the data; K.K., K.T., H.T., and F.S. contributed to the development of the experimental setup; H.I. wrote the paper.

Funding: A part of this work was supported by the Tateisi Science and Technology Foundation.

Conflicts of Interest: The authors declare no conflict of interest.

\section{References}

1. Hasegawa, K.; Qiu, L.; Shinoda, H. Midair Ultrasound Fragrance Rendering. IEEE Trans. Vis. Comput. Gr. 2018, 24, 1477-1485, doi:10.1109/TVCG.2018.2794118.

2. Dobbelstein, D.; Herrdum, S.; Rukzio, E. inScent. In Proceedings of the 2017 ACM International Symposium on Wearable Computers, Maui, HI, USA, 11-15 September 2017; pp. 130-137, doi:10.1145/3123021.3123035.

3. Narumi, T.; Nishizaka, S.; Kajinami, T.; Tanikawa, T.; Hirose, M. Meta Cookie+: An Illusion-Based Gustatory Display. In Virtual and Mixed Reality-New Trends; In Proceedings of the Virtual and Mixed Reality 2011, Orlando, FL, USA, 9-14 July 2011; pp. 260-269, doi:10.1007/978-3-642-22021-0_29.

(C) 2018 by the authors. Licensee MDPI, Basel, Switzerland. This article is an open access article distributed under the terms and conditions of the Creative Commons Attribution (CC BY) license (http://creativecommons.org/licenses/by/4.0/). 